\title{
Implementing Stack E6 via OS Linux Sockets
}

\author{
Zaitsev D.A., Kharsun M.A. \\ Dr.Sci., Professor \\ Senior Member of the IEEE, Member of ACM, SIAM \\ International Humanitarian University \\ E-mail: zsoftua@yahoo.com \\ Bachelor of Computer Science \\ International Humanitarian University \\ E-mail: mikefromsky@gmail.com
}

\begin{abstract}
New software implementation of Ukrainian national stack of networking protocols E6 was presented. Within operating system Linux kernel, families of E6 protocols and addresses were created as well as functions of their processing, in the form of a loadable module. Application of socket technology gives a series of considerable advantages among which unified with other stacks application interface, reuse of the kernel resources facilitating the further development and enterprise implementation of stack E6, engineering corresponding networking devices.
\end{abstract}

Keywords: stack of networking protocols, E6, socket, kernel, Linux

\section{Introduction}

Packet switching networks dominate in modern communications world. There is a tendency of network devices transformation into specialized computers with predominance of software that implements high layers of open systems interconnection basic reference model before data-link encoding and direct transfer of signals within a media.

Acquirement of specialized software development methods for the implementing network protocol stacks, packet switching and routing algorithms is crucial for providing ways not only to exploit devices that are developed in other countries, but also to produce the own ones. 
Recently Ukraine turns into a country, using network technologies developed in other countries, due to the importing network devices and passive equipment. Not only development, but the production of high-tech equipment is situated outside the country.

Information about using programming code in the shape of virus-worm for destabilization of uranium enrichment centrifuges in Iran appeared in ACM news. That's why aspects of exploited software source code openness become more important. Complexities in examination of open specifications stimulate own development of hardware (processors) by many other countries. Well known examples of using software and hardware methods of communicating equipment in realization of military and political sanctions against Third World countries promote the initiation of national programs of production computing and communications machinery.

As the production of equipment requires significant investment, the software development is a more dynamic sphere. Its possibility of expansion is stipulated by personnel's level of qualification and organization. Using operating environments compatible with freeware OS Linux in network devices, allows an experimental development on home and office personal computers.

In Ukraine, a national stack of networking protocols E6 [1,2] was developed and provided its experimental realization via additional OS Linux kernel system call $[3,4]$. In spite of some advantages, said way has significant number of shortcomings, which prevent further development of national stack E6 and its industrial implementation. The main flaw is the need of OS kernel recompilation for the new system calls addition and substantial modification of the API; besides there are no possibilities of the reuse kernel methods involved in the implementation of other stacks, TCP/IP for example.

Therefore, the independent realization of E6 datagram style on the basis of standard OS Linux socket interface (the same as vast majority of other well known protocols) was implemented in International Humanitarian University (www.mgu.com.ua). This development is unique, because it requires a detailed study of OS Linux kernel environment and significant integration with its data structures and functions.

The goal of the present work is the rendering the software implementation of E6 stack via OS Linux socket interface. It may be also considered as a case study of new protocols software implementation in Unix-like operating environments.

\section{Creating E6 sockets within OS Linux}

OS Linux socket is a generalized inter-process communication channel, which is represented as a file descriptor. As a rule, sockets are used for communication between processes running on different computers. To provide communication between two sockets, they have to support the same style and 
protocol of interaction. There are three basic styles supported: datagram (DGRAM), stream (STREAM) and raw (RAW).

For the external indication of socket, its address is used, so the main features of socket classes are address family $(A F)$ and the appropriate protocol family $(\mathrm{PF})$. Among the known address families, AF_LOCAL - local format of operating system and AF_INET - IP address family should be noted. During the realization, the new E6 address family was created.

The main advantage of using sockets is a standard and consistent API for each protocol, implemented via this interface. Basic functions of sockets are contained in libc library, the main header file is sys/socket.h; besides for specific address/protocol families, own header files are added, netinet/in.h in TCP/IP, for example. For stack E6, the file e $6 . \mathrm{h}$ was created.

Socket is created by the function

int socket (int protocol family, int style, int protocol)

as result, the integer descriptor of associated file is returned; the set of available protocols is determined by the selected family, for example, UDP_PROTO, TCP_PROTO for TCP/IP family. In most cases, the protocol is uniquely determined by the chosen style and can be omitted.

E6 socket for the datagram communication mode, that is similar to UDP protocol, is created by the command

sock = socket $($ PF_E6, SOCK_DGRAM, IPPROTO_UDP $)$;

Binding socket with a particular address is performed by the function

int bind (int socket, struct sockaddr *addr, socklen_t length) where sockaddr structure is a generic data type, which allows using different address systems, so the third parameter is an actual length of the structure. For E6 address family the following description from $e 6 . \mathrm{h}$ header file is used

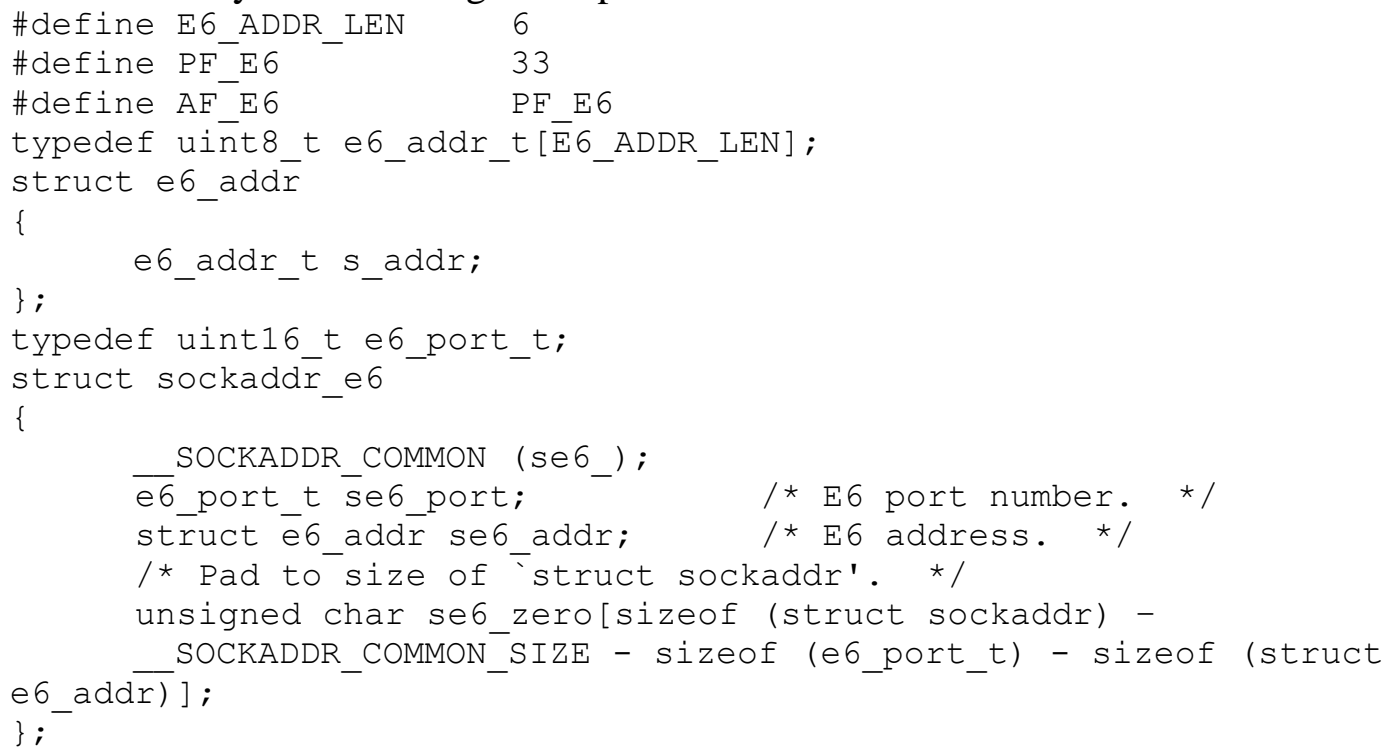

Thus E6 socket address sockaddr_e 6 consists of E6 host address of 6 octets and E6 port number of 2 octets. System of addresses and ports is 
independent of other protocol families; particularly, it is independent of TCP/IP (fig. 1).

An example of binding E6 server socket that allows calls from arbitrary E6 addresses to port 25 , has the following form

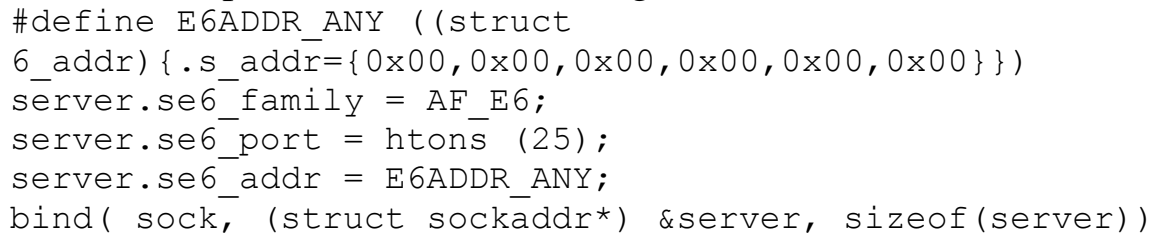

Note, that operation of conversion (struct sockaddr*) results in the transforming a pointer of specific socket address to a generalized type, that is required by function bind; zero address $E 6 A D D R \_A N Y$ can receive datagrams from arbitrary E6 addresses.

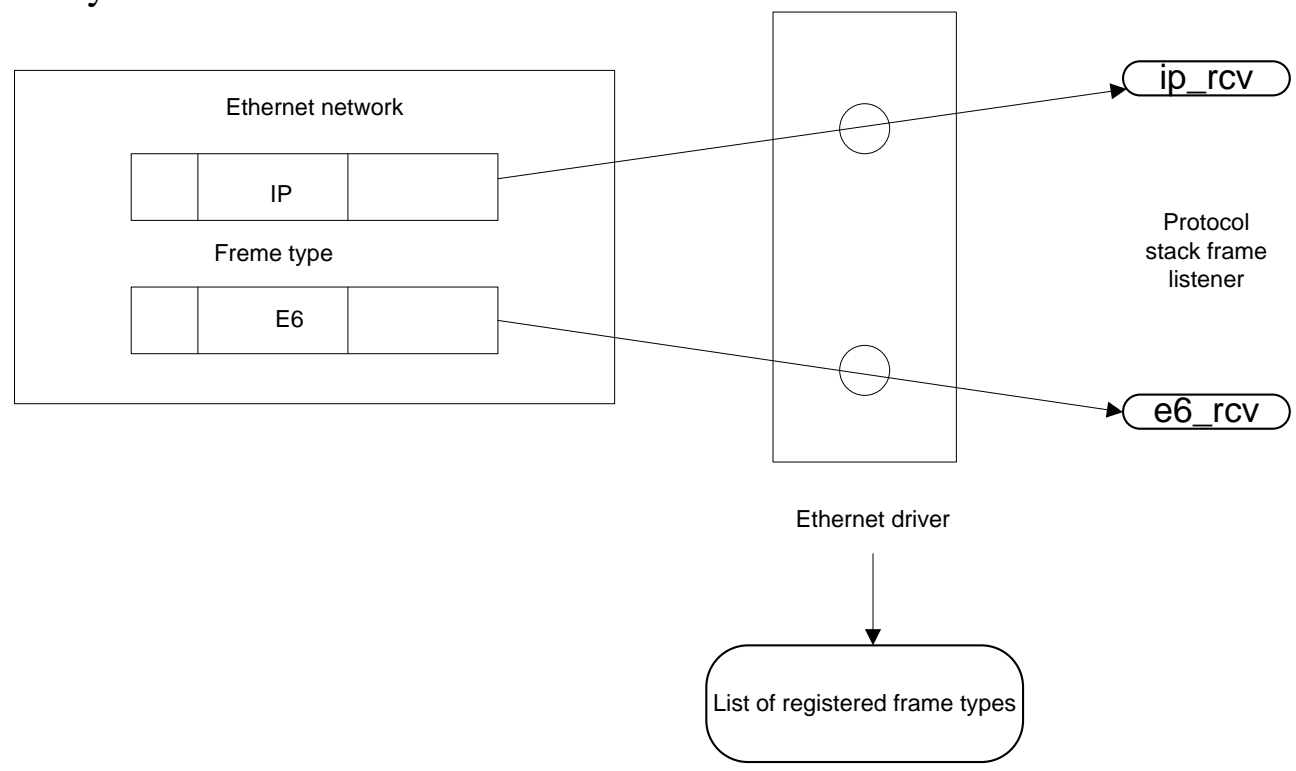

Fig. 1: Demultiplexing frames of different types by Ethernet driver

After binding, the exchange of data is performed by recvfrom and sendto functions, which provide an opportunity of remote socket address receiving. In addition, it is permissible to establish a connection with connect function and then the further exchange is performed by functions send and recv, which do not require address of the remote socket; this style of exchange is more characteristic for the stream mode.

Functions' headers look as

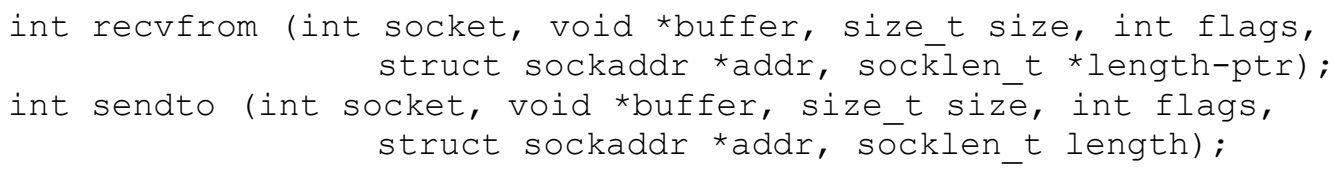

where buffer variable points to the datagram address buffer, and size is its length in bytes, flags variable sets the mode of exchange and in the simplest case equals to zero. Functions return the actual number of sent/received bytes; negative 
values correspond to an error. Function recvfrom creates socket address addr and length values using length-ptr pointer in accordance with the actual sender of the datagram; sendto function requires the receiver socket address addr and its length length.

An example of the server work cycle body has the form

len = recvfrom ( sock, request, 1500, 0, (struct sockaddr *)\&client, \&size );

<обработка запроса request, формирование ответа reply>

len = sendto ( sock, reply, len, 0, (struct sockaddr *)\&client, size );

The socket address mapping into domain names is also provided by gethostbyname function and its modifications as well as socket listening listen and receiving connection requests accept. These methods are not used in the current implementation of E6 stack.

\section{Interface of the loadable kernel module E6-socket}

Almost each OS Linux protocol stack is implemented as a loadable kernel module. On the one hand it provides some autonomy for independent development, and on the other hand the direct use of the kernel environment in the form of special data structures and functions. Some modules, such as, for example, TCP/IP stack, is loaded together with the OS loading that creates an external effect of its presence in the static part of kernel - binary file linuz.

Modules that are not included in Linux startup scripts are loaded, unloaded and maintained by special commands:

insmod <module name> <parameters>-load module;

rmmod <module name>- unload module;

modinfo <module name $>$ - get information about loaded module;

modprobe <keys > <module name> <parameters $>$ - load system module.

Parameters are formed by special macros module_param during the module development and have the following key format of instruction

$<$ parameter name $>=<$ parameter value $>$.

Command insmod loads an arbitrary specified file, whereas command modprobe works with modules located in $/ \mathrm{lib} /$ modules directory and registered within system with depmod command; advantage of modprobe command is the ability to specify additional keys.

E6-socket module has name e6.ko; type .ko is standard and formed from the reduction of words "kernel object". To build a kernel module, a special way of compilation and linking with make command is used to access the current kernel symbol tables; the required by command make files makefile and kBuild are the following:

Makefile: :

obj-m :=e 6.0

KERNELDIR $:=/ 1 \mathrm{ib} / \mathrm{modules} / 2.6 .31 .5$-desktop-1mnb/build

all:: \$ (MAKE) -C \$ (KERNELDIR) M=`pwd' modules 
KBuild: :

obj-m : $=e 6.0$

E6-socket module parameters are: name of E6 device in OS Linux devices' name format - E6 devname, E6 device address (put in place of the factory MAC-address) - E6_devaddr. Loading command example is the following:

insmod e6.ko E6 devname=eth0 E6 devaddr $=000000000001$

In case of conflict when loading the module into the unknown kernel, using the modprobe command with the forced loading keys is recommended.

After E6-socket module is loaded, it is integrated into the system of known protocol stacks and Ethernet frames by registering new protocol family $\left(\mathrm{E} 6 \_\mathrm{PF}=33\right)$ and new frame type $\left(\mathrm{ETH} \mathrm{P}_{-} \mathrm{E} 6=0 \times \mathrm{E} 600\right)$. As a result, the standard socket environment sets pointer to E6 create socket program e6_create, and the Ethernet driver receives a pointer to E6 frames processing program e6_rcv.

This provides the use of standard user interface to work with sockets' library libc, as described in the previous section, based on the functions socket, bind, recvfrom, sendto, and others. Necessary supplements are associated with a specific E6 address format, which determines the appropriate E6 socket format. Recall that, in comparison with the IP address, the E6 address has a length of 6 bytes, the hierarchical structure and is used both as a network and the datalink address at the same time [1,2] - instead of the IP and MAC addresses.

Data structures for describing the E6-address, E6-socket, and macros for standard E6 addresses, such as the loopback address, arbitrary address, and broadcast address are collected in the customer header file e6.h. Said header is required to specify in the include directive in the beginning of an application that uses the E6-socket module facilities:

\#include "e6.h"

For testing the E6-socket module, the simplified remote shell application was used. It is recommended for self-study training to modify and recompile the standard TCP/IP stack applications, for example, TFTP to work via E6 stack.

\section{The interaction of E6-socket module with the kernel}

The interaction of a loadable module with the kernel is divided into four main classes:

- call to the module initialization function;

- call to the module deinitialization function;

- calls of the kernel to the module standard functions for working with E6 sockets;

- calls of the module programs to the supporting functions of the kernel.

The overall structure of the E6-socket module is shown in fig.2. After the module is loaded into memory, the insmod command starts automatically the module initialization function that is registered with the system macro

module_init (e6_init); 
the header of the initialization function has the following form static int init e6_init (void)

Within the initialization function, the following procedures are executed: E6 protocol family registering, E6-device search and its initialization and the announcement of a new type of E6 frame. Thus the loadable module methods are integrated into OS Linux kernel environment. To register a family of protocols, the function

proto_register(\&e6_proto, 0);

is called which receives the e6_proto structure address indicating the name, owner, and the length of E6 protocol family socket. Structure e6_proto has the following form

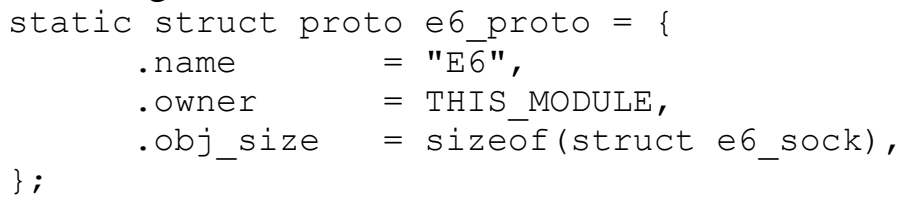

Then the E6-device initialization takes place by the internal E6-socket module function e6_init_dev call

e6_dev=e6_init_dev (e6_devname, e6_devaddr, \&e6_myaddr);

During executing this function, an attempt of the network device initialization is done; in case of success E6 address is assigned to the network device and the system is notified regarding the readiness of the device to the further work. Then the virtual loopback interface is initiated

lo_dev = dev_get_by_name (\&init_net, lo_devname) ;

The next command

sock_register(\&e6_family_ops);

implements the E6 socket registration: it specifies the protocol family, address of the sockets' creating function and their owner. Structure e6_family_ops has the following form:

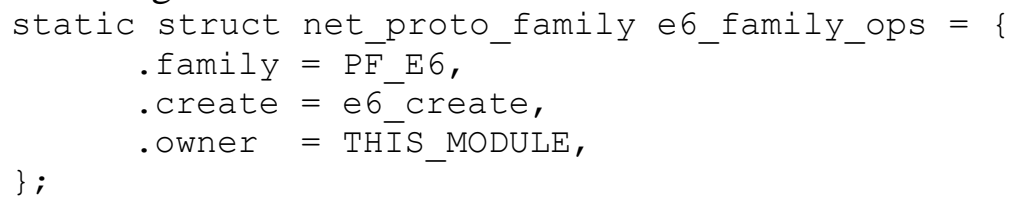

It contains E6 protocol family name, function e6_create to create a socket of this type and its belonging. Function e6_create has the header

static int e6_create (struct nēt *net, struct socket *sock, int protocol)

and describes the process of creating E6 socket.

The following call of function

dev_add_pack (\&e6_packet_type) ;

registers new type of Ethernet frame: this function adds specified frame type handler, using e6_packet_type structure and includes E6 frame type into the kernel database

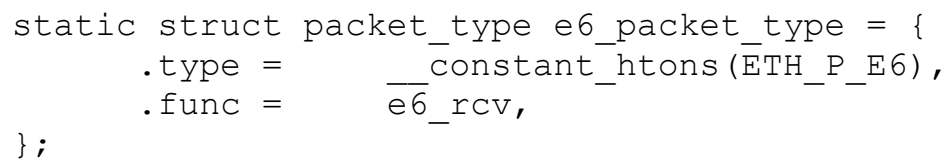




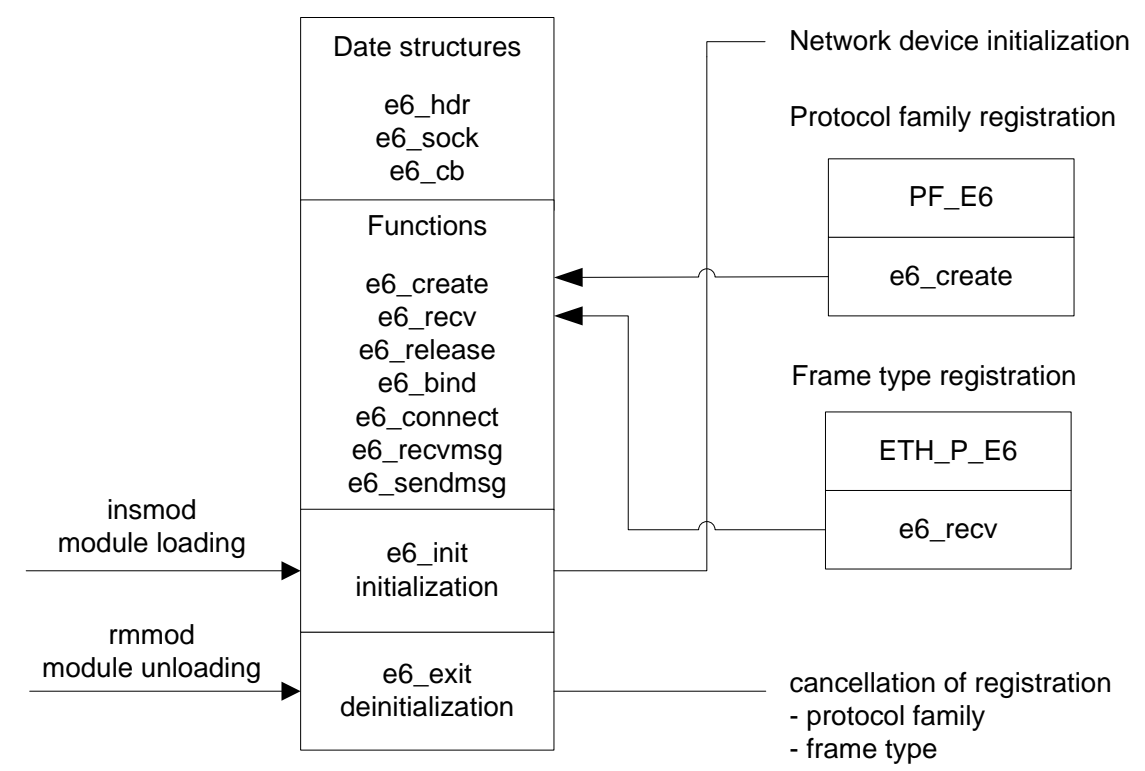

Fig. 2: E6 loadable module structure

As a result of executing the loadable module initialization function, a set of data structures, constants, macros and functions joins OS Linux kernel, to implement the datagram mode of transmission on the basis of the E6 stack. There are two basic module entry points that are imported into OS Linux database: e6_create to create E6 socket with socket user-end function at protocol family PF_E6 specification; e6_rCV for the listening received frames of ETH_P_E6 type. Thus, module interfaces of application and data-link layers are created.

Access to the functions of E6 module, implementing the standard socket interface (bind, connect, release, recvfrom, sendto) is provided by the table e 6 dgram ops of pointers to the standard socket entry points

const struct proto_ops e6_dgram_ops $=\{$

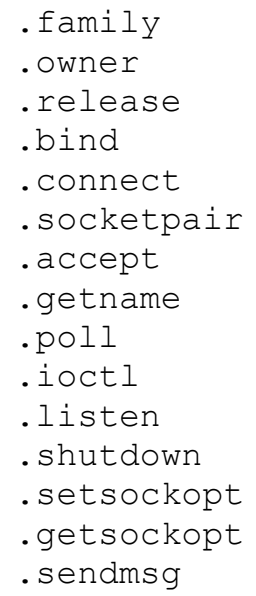




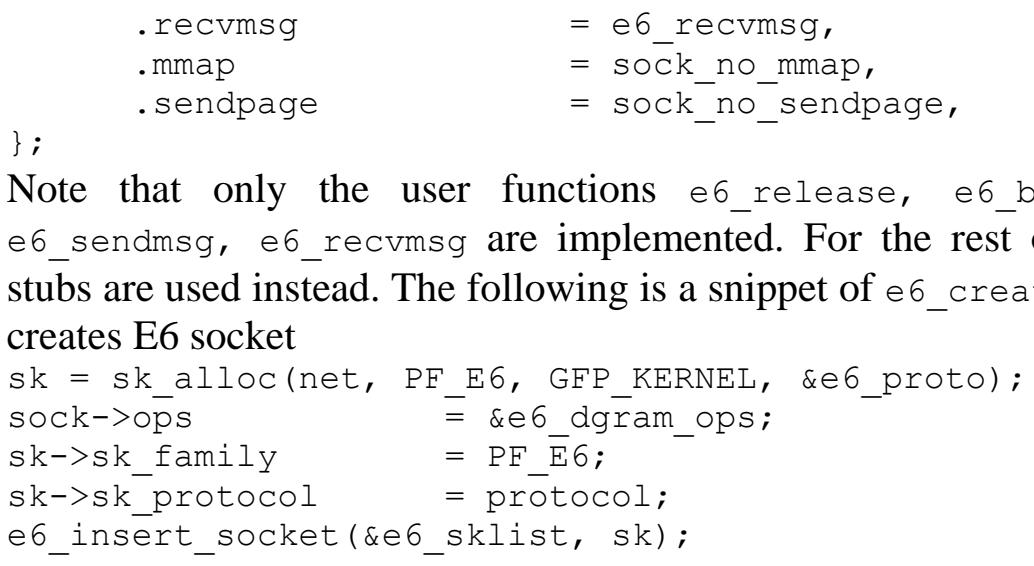

After the memory allocation by sk_alloc kernel function, address of table of pointers to the standard functions sock->ops is installed. So each socket contains a pointer to the table of its functions, which provides its autonomy. Finally, created socket is included into general E6 socket list by e6_insert_socket function.

In the case of input rmmod command from the Linux console, the process of loadable module deinitialization begins. Before releasing memory occupied by the module, the entry point to the completion of the module is automatically invoked, given by the system macro

module_exit (e6_exit);

The header of the completion function of the module has the form static void exit e6 exit (void)

The main purpose of this function is cancelling the registration of the protocols family and types of packets (frames). The function call

dev_remove_pack (\&e6_packet_type);

removes records about E6 frame type in the kernel database. Then functions

sock_unregister(PF_E6);

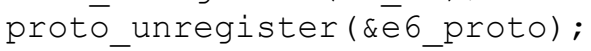

perform removal of E6 protocol and addresses family from the kernel database.

Thus, the two entry points to the module are explicitly registered and called by the kernel in the following cases: e6 create to create a customer E6 socket with the socket function; $e 6_{-} r \mathrm{cv}$ by Ethernet driver when receiving E6 frames. Other functions correspond to the user-end functions to work with sockets; kernel determines their addresses from e6_dgram_ops entry points table for the specified socket (fig.3). 


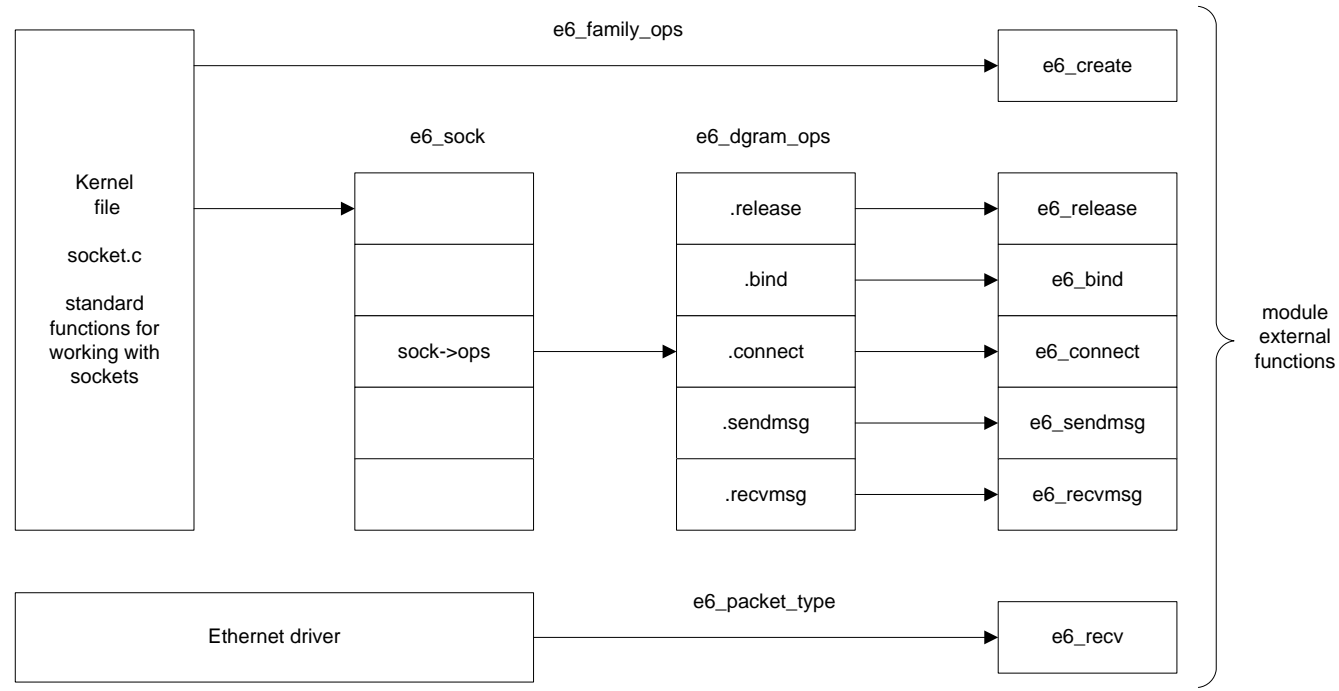

Fig. 3: Access to the E6-socket module functions

More subtle issues of interaction, which reveal the main stages of the transfer of control and data from the application process and the network device driver by the module functions, are discussed in Section 6 .

\section{Algorithms of E6-socket module basic functions}

To understand the algorithms of the module, should pay attention to the basic data structures at first. In addition to described in the previous section structures e6_proto, e6_family_ops, e6_packet_type, providing the registration of a new stack in the kernel, as well as structures sockaddr_e6, e6_addr and the type e6_port_t used to specify the E6 addresses and described in Section 2, the module contains a description of a number of internal data structures.

Moreover, the module uses the standard kernel data structures

struct socket-generalized socket;

struct sock - representation of a socket at the network level;

struct sk_buff - buffer for the transmission of the Ethernet frame;

struct net_device-network device descriptor.

Generalized socket is designed to provide a uniform standard handling of different types of sockets in the kernel; structure socket contains a pointer to the associated structure sock; frame buffers sk_buff are created by the module during message transmission and by driver during receiving frame from network; net device descriptor net_device contains its features. To understand the interaction between data structures, it is essential to the study formed linked lists. All E6 sockets are collected in a list e6_sklist, in addition, all the sockets of all 
types are included in the general list. To each socket sk, a queue of received messages of sk_buff type is formed. To network device net_device, a single queue of sent packets (frames) of type sk_buff is formed. Note, that the key of socket search in e6_sklist list is E6 port.

Thus, the message transmission function comes to the formation of sk_buff and inserting it to the queue of network device with net_device header; the receiving message function comes to the extracting a frame from the appropriate socket queue sk, depending on specified flags in case of the frame absence in the queue, either the blocking of the process is executed for frame entrance waiting or an error code returning. Frames sk_buff get into the socket queue sk via the call by Ethernet driver of registered during module initialization function e6_rcv after receiving E6 frame from network.

Module contains definitions of the following internal data structures. E6 frame header (data-link and network) contains pares of E6 addresses of receiver de $6 \mathrm{a}$ and sender se $6 \mathrm{a}$, and pares of sender de $6 \mathrm{p}$ and receiver se $6 \mathrm{p}$ ports struct e 6 hor \{

$\begin{array}{ll}\text { struct e6_addr } & \text { de6a; } \\ \text { struct e6_addr } & \text { se6a; } \\ \text { be16 } & \text { type; } \\ \text { e6_port_t } & \text { de6p; } \\ \text { e6_port_t } & \text { se6p; }\end{array}$

\};

E6 socket contains standard socket sk and specific fields to specify the E6 addresses and port numbers of the sender saddr, sport and receiver daddr, dport and flags as well.

struct e6_sock \{

strüct sock sk

struct e6 addr saddr;

e6_port_t ${ }^{-}$sport;

struct é6_addr daddr;

e6_port_t ${ }^{-}$dport;

un16 cmsg_flags;

\};

The following static variables are created

static struct hlist_head e6_sklist; - header of E6 sockets list;

struct e6_addr e6_myaddr; - own E6 address;

static struct net_device ${ }^{*} 6$ _dev; - address of E6 device descriptor.

Let us classify the functions of E6-socket module:

- initialization and deinitialization functions e6_init and e6_exit;

- socket user API implementation functions: e6_create, e6_release, e6_bind, e6_connect, e6_sendmsg, e6_recvmsg;

- function-handler of received E6 packets (frames) e6_rcv;

- supporting functions: socket removing e6_remove_socket, inserting socket to the list e6_insert_socket, searching socket with specified port e6_find_port, searching free port e6_bind_port, searching socket with 
specified address and port e6_find_socket, device initialization e6_init_dev.

All module functions and data structures have prefix e $6_{-}$. Note that for the implementation of several user functions, only one module function can be used; for example to implement functions sendmsg, sendto, send in the datagram style, the module function e6_sendmsg is used, and to implement recvmsg, recvfrom, recv functions, é 6 recvmsg is used. Formation of various call parameters is executed by intermediate kernel programs that are located in socket.c kernel file.

E6 socket creating function code snippets were considered in Section 4. Let us study the functions' algorithms implementing sending and receiving message operations. The sending message function has the form

static int e6 sendmsg(struct kiocb *iocb, struct socket *sock, struct msghdr ${ }^{*} m s g$, size_t len)

where iocb is the block of input/output control, msg is a message header, len is the message length. Initially, memory is allocated for the packet (frame) buffer

$\mathrm{skb}=$ sock_alloc_send_skb(sk, len + sizeof(struct e6_hdr), msg>msg_flags \& MSG_DONTWÄIT, \&err);

Copying information from the user's buffer to skb block is performed by the memcpy_fromiovec command using the message header msg parameters skb reserve (skb, sizeof (struct e6 hdr)); $\operatorname{err}{ }^{-}=$memcpy fromiovec (skb_put (skb, len), msg->msg_iov, len); initial part of buffer is reserved by skb_reserve command for subsequent placement of e6 har header. Recall that, e 6 hdr is a combined network and datalink layers header. Then the packet (frame) header is formed

hdr $=$ (struct e6_hdr *) skb_mac_header (skb);

hdr $->\operatorname{de} 6 \mathrm{a}=$ daddr;

hdr $->\operatorname{se} 6 \mathrm{a}=\mathrm{addr}$;

hdr->type = htons (ETH_P_E6);

hdr->de6p = dport;

hdr->se6p = port;

The output device and the priority of the operation is specified

$\mathrm{skb}->\mathrm{dev}=$ e6_dev;

skb->priority ${ }^{-}=$sk->sk_priority;

The actual launch of the operation on the device is performed by the sequence of commands

dev queue xmit (skb);

dev_put (deve);

Command dev_queue_xmit inserts the frame buffer skb to the device queue; command dev_put forces the device driver to get started in case of absence of active operation and does not involve any changes otherwise; the driver will check the queue at the end of the active operation.

Message receiving function has the header

static int e6 recvmsg(struct kiocb *iocb, struct socket *sock, struct msghdr ${ }^{*} \mathrm{msg}$, size $t$ len, int flags)

where the assignment of parameters corresponds to the previously described function e6_sendmsg reversing the transmission direction at the data 
interpretation. Getting datagram in skb block from queue to the specified socket sock is performed by the command

skb=skb_recv_datagram (sk, flags, flags\&MSG_DONTWAIT, \&err);

which in case of the datagram absence blocks the current process at indicating MSG_WAIT flag and returns an error at MSG_DONTWAIT flag. Then the copying of received data from skb block to user buffer is done, which msg->msg_iov address is contained in msg header block

err $=$ memcpy_toiovec (msg->msg_iov, skb->data+4, copied);

Then from skb block the socket address is copied to the to message header block msg for the following return to the application process

msg->msg_namelen $=$ sizeof (struct sockaddr_e6);

memcpy (msg->msg_name, skb->cb, msg->msg_nämelen);

Using a simple copy command memcpy is due to the fact, that copying the socket address is performed inside the kernel address space as opposed to copying data from kernel space to user space (input/output block) by command memcpy_toiovec. The processing is completed via releasing datagram memory by the command

skb_free_datagram(sk, skb);

Thus, the receiving function is related to network device driver asynchronously through a datagram queue skb to a socket, which is formed by e6_rcv function considered later.

Function of handling E6 frames received by driver has the header

static int e6_rcv(struct sk_buff *skb, struct net_device *dev, struct packet_type *pt, strüct net_device *orig_dēv)

where skb is a received frame buffer, dev is a network device, pt is a frame type descriptor, orig_dev is a source device, which may not match the dev in the case of redirection.

Function e6_rcv is called by Ethernet driver after receiving frame of type $\mathrm{ETH}_{-} \mathrm{P}{ }_{-} \mathrm{E} 6$ in accordance with registered during initialization packet type e6_packet_type, address of the corresponding structure pt is specified additionally in call parameters.

The driver performs a preliminary check of the packet destination address, the results of which can be used to ignore packets addressed to other hosts, because E6 address is used as well as the MAC address and set on the NIC during the module initialization

if (skb->pkt_type == PACKET_OTHERHOST) goto drop;

Label drop designates action of the packet ignoring, reduced to releasing block skb and return of the error code NET_RX_DROP

drop: kfree_skb(skb);

return NET_RX_DROP;

In the module, a reliable version of the test is used in the form of an explicit comparison of E6 destination address with own e6_myaddr and loopback lo e6 addr addresses`

hdr $\bar{r}=$ (struct e6_hdr *) (skb->data-E6_TRANSPORT_HEADER_OFFSET);

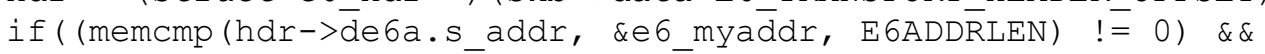



drop;

(memcmp (hdr->de6a.s_addr, \&lo_e6_addr, E6ADDRLEN) !=0) ) goto

Then the search for E6 socket with specified in the datagram port is run sk = e6_find_socket (hdr->de6p, hdr->de6a);

if (!sk) goto drop;

Then the socket address of the received frame is stored in the supporting block cb for the further use in the receiving packet function e6_recvmsg

e $6 \mathrm{cb}=$ (struct e6 cb *) skb->cb;

e $6 \mathrm{cb}->\operatorname{se} 6$. se 6 fämily=AF_E 6 ;

e $6 \mathrm{cb}->\operatorname{se} 6 . \mathrm{se} 6$ - $a d d r=h d r-\overline{s e} 6 \mathrm{a}$;

e6cb->se6.se6_port=hdr->se6p;

The processing is completed with inserting skb block to the queue of received datagrams for found socket $\mathrm{sk}$

if (sock_queue_rcv_skb(sk, skb)) goto drop;

Nonzero return code of sock_queue_rcv_skb function corresponds to an error and leads to ignoring the packet. Interaction of Ethernet driver, e6_rcv function and e 6 recvmsg function are illustrated in fig.4. Note that running of functions e6_rcv, e6_recvmsg is asynchronous: e6_rcv is called by driver and e6_recvmsg runs while handling the corresponding system call of the application process.

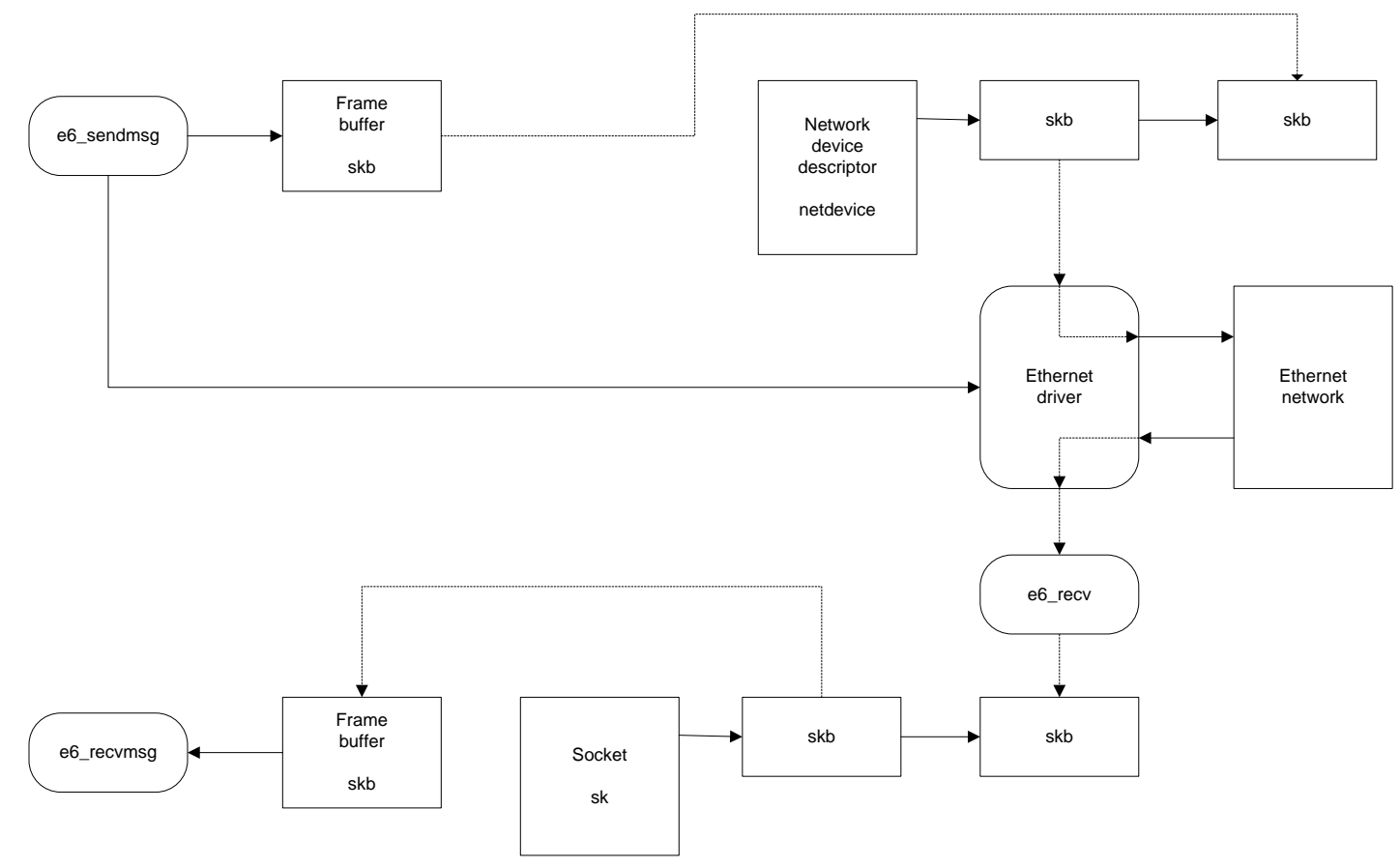

Fig. 4: Implementation of the datagram send/receive functions 


\section{Tracing E6 packets transfer processes}

Tracing the sequence of actions implementing certain user commands allows us to understand peculiarities of the interaction among:

- application source code in $\mathrm{C}$ language written by the user;

- the object code of the library libc linked to the application;

- static part of the Linux kernel;

- loadable module.

Let us consider the tracing process on the example of user-defined function of the receiving message by a server application

len = recvfrom ( sock, request, 1500, 0, (struct sockaddr *) \&client, \&size );

Initially, control inside application code is passed into recvfrom function of $l i b c$ that performs a preliminary check of parameters and generates a block of input/output parameters presented with an array of type long.

Then it calls the syscall function of $l i b c$ with the number of system call SYS SOCKETCALL=102 and the number of subfunction call=SYS RECVFROM=12, which execution leads to the application processor interruption number $0 \times 80$, intended to implement the kernel system calls in Linux.

From the interrupt vector $0 \times 80$ in operating memory a new processor status word is retrieved, which contains the system call handler address system_call, and performs switching operation of the processor from the user to the system mode (fig. 5).

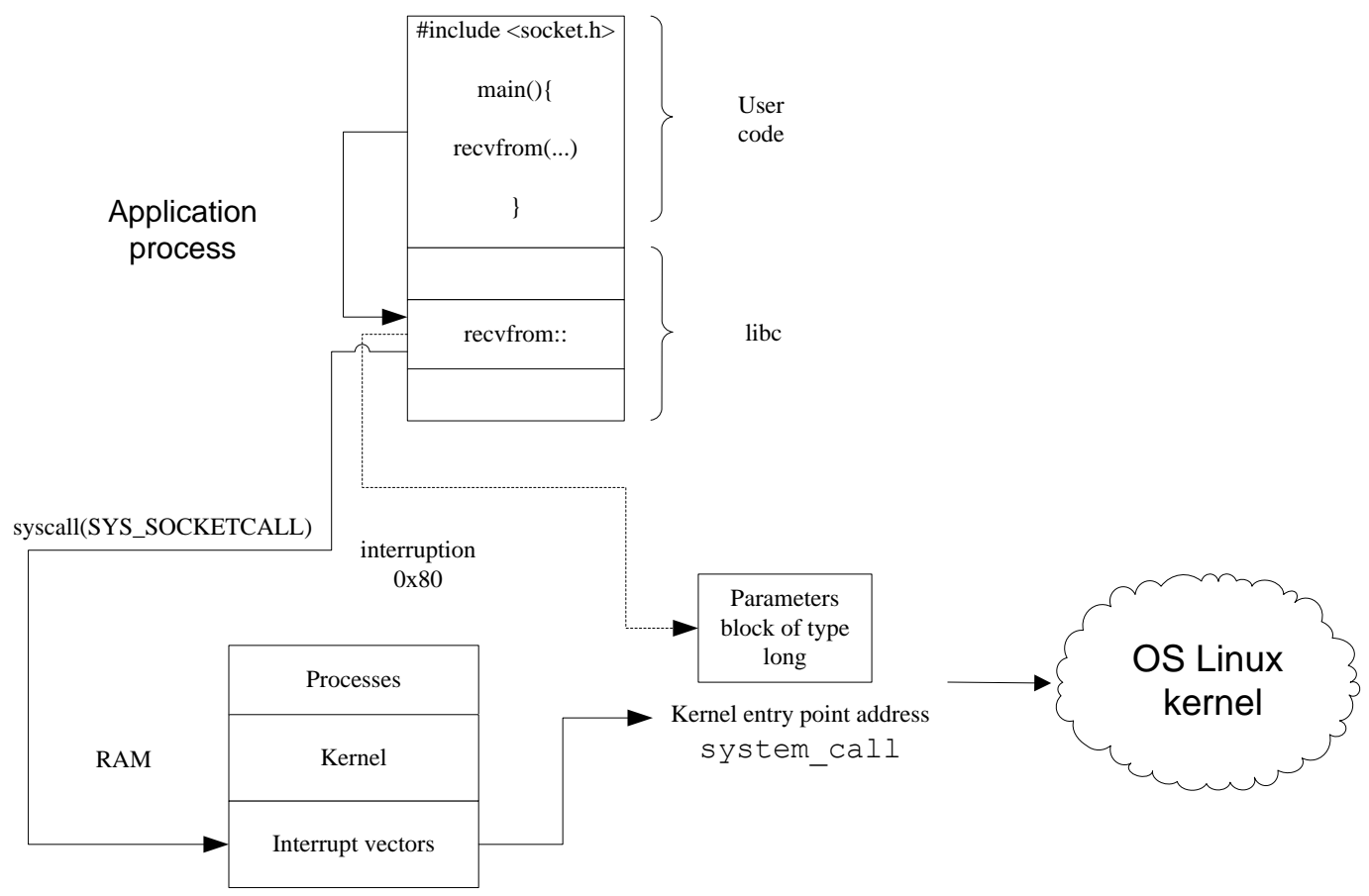

Fig. 5: Sequence of entry into OS Linux kernel 
The program system_call placed in the kernel file arch/x86/kernel/entry_64.S works as a manager (switch): it finds the record having number SYS SOCKETCALL $=102$

.long sys_socketcall

in system call table sys_call_table of kernel file syscall_table_64.s that contains the address of the function sys_socketcall which is a handler of all system calls regarding sockets.

Program sys socketcall is a manager of socket system call subfunctions, which retrieves the parameters from the parameters block and passes control to the appropriate subprogram.

For SYS_RECVFROM subfunction, subprogram sys recvfrom is called err = sys_recvfrom(a0, (void_user *)a1, a[2], a[3], (struct sockaddr _user *)a[4], (int_user *)a[5]);

which receives the first six parameters from the parameters block in accordance with previously described format of the user-end function recvfrom; for addresses specified by the user, user address space indicator _ user is written explicitly.

The header of sys_recvfrom program is formed by the system macro SYSCALL_DEFINE6 (recvfrom, int, fd, void_user *, ubuf, size_t, size, - unsigned, flags, struct sockaddr _user *, addr, int user *, addr len)

which contains sequentially the types and names of parameters after the function name. First of all, the address of socket in memory is determined on the file descriptor, which the operation is performed for

sock = sockfd_lookup_light (fd, \&err, \&fput_needed);

Then the call parameters are copied into the fields of the message header block msg of type msghdr and an input/output control unit (vector) iovec of type iov

msg.msg_control = NULL;

$\mathrm{msg} \cdot \mathrm{msg}$ controllen $=0$;

msg.msg iovlen $=1$;

$\mathrm{msg} \cdot \mathrm{msg}$ iov $=$ \&iov;

iov.iov_len = size;

iov.iov_base = ubuf;

msg.msg_name $=$ (struct sockaddr *) \&address;

msg.msg_namelen = sizeof (address);

Then the program sock_recvmsg is called

err = sock_recvmsg (sock, \&msg, size, flags);

The program sock_recvmsg after operations of safety audit is completed by the calling

sock->ops->recvmsg(iocb, sock, msg, size, flags);

thus, the address specified by the description line

. recvmsg = e6_recvmsg,

is retrieved from e6_dgram_ops structure, which leads to the actual e6_recvmsg function call of loadable module E6-socket (fig. 6), which algorithm was studied in the previous section. 


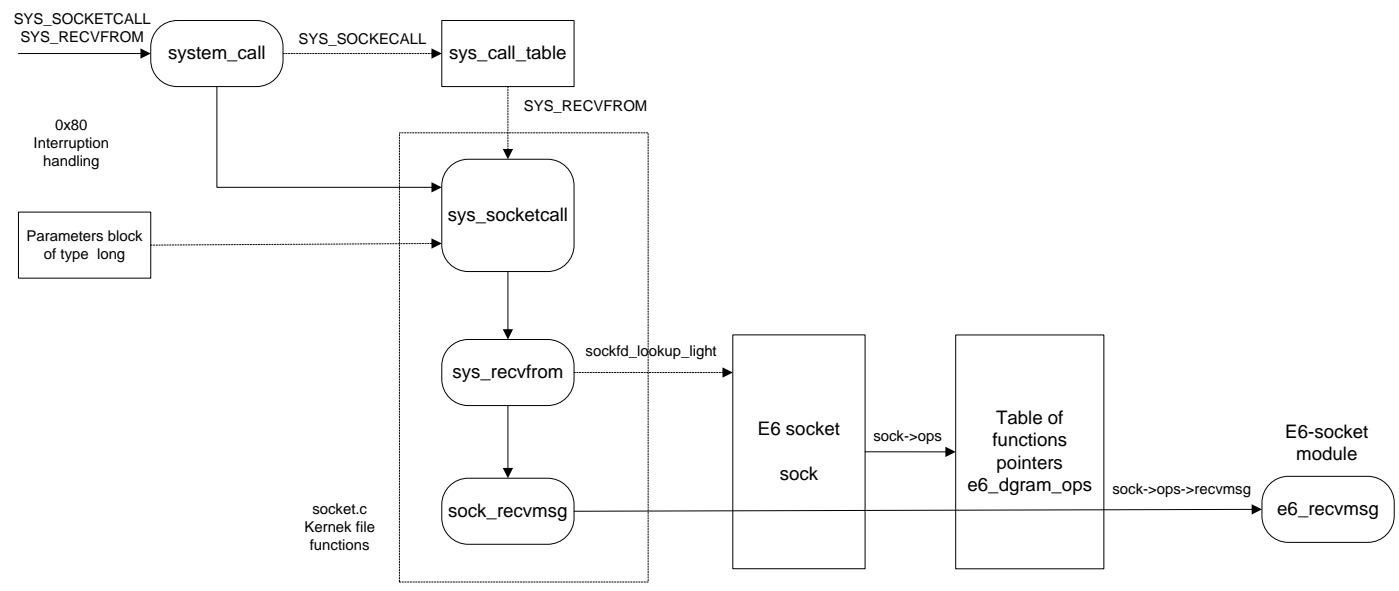

Fig. 6: The sequence of the module function calls within the kernel

Note that after the system call completion the reverse sequence of actions in not obligatory. Some of calls result in blocking the current process, for example, waiting for a message from the network. During this time, the kernel starts to execute other ready-to-execute processes, or a special process-sloth idle in the absence of ready processes.

In this case the change of process status will be caused by NIC interrupt and after its completion the driver starts the program e6_rcv, which in turn changes the state of the process while inserting the frame into the queue to the appropriate socket.

\section{Conclusion}

The technology of software implementation of Ukrainian national protocol stack E6 (the datagram mode) via OS Linux kernel sockets was presented which allows minimizing modification of user interfaces and provides the kernel code reuse that significantly reduces the complexity of development and creates a prototype for further industrial implementations of stack E6. The implementation was done in the kernel version 2.6.31.5 of the operating system Linux Mandriva 2010 and then moved to Linux Ubuntu, Debian, Fedora.

The material can also be considered as a case study of the software implementation technology of new protocol stacks in Unix-like operating environments.

The source files of the kernel module and test applications with brief instructions on how to compile, install and run them are put on the website http://daze.ho.ua and can be used as prototypes for the programmers to develop their own protocol stacks. 


\section{References}

[1] Zaitsev D.A., Bolshakov S.I. "E6 Addressing Scheme and Network Architecture" Journal of Advanced Computer Science and Technology, No. 1, (2012), pp.18-31.

[2] Vorobiyenko P.P., Zaitsev D.A., Nechiporuk O.L. "World-wide network Ethernet?” Zviazok (Communications), No. 5, (2007), pp.14-19. In Russ.

[3] Zaitsev D.A., Guliaiev K.D. "Stack E6 and its Implementation within Linux Kernel" Journal of Software Engineering and Applications, No. 4, (2011), pp.379-387.

[4] Guliaiev K.D., Zaitsev D.A. "Experimental Implementation of Networking Protocols Stack E6 into OS Linux Kernel” Artificial Intelligence, No. 2, (2009), pp.105-116. In Russ.

[5] Herrin G. Linux IP Networking, TR-0004, (2000).

[6] Bovert D., Cesati M. Understanding the Linux Kernel, O'Reilly, (2000). 